\title{
On the use of multicriteria decision analysis to formally integrate community values into ecosystem-based freshwater management
}

Simone D. Langhans 1 https://orcid.org/0000-0001-9581-3183, Sonja C. Jähning 1 https://orcid.org/0000-0002-63499561, Marc Schallenberg

DOI

https://doi.org/10.1002/rra.3388

Original publication date

05 December 2018

\section{Document version}

Accepted version

\section{Published in}

River Research and Applications

Citation (Vancouver)

Langhans SD, Jähnig SC, Schallenberg M. On the use of multicriteria decision analysis to formally integrate community values into ecosystem-based freshwater management. River Research and Applications. 2018. 
1 TITLE: On the use of multi-criteria decision analysis to formally integrate community values

2 into ecosystem-based freshwater management

4 RUNNING TITLE: Community values in freshwater management

6 Simone D. Langhans ${ }^{1,2,3^{*}}$, Sonja C. Jähnig ${ }^{3}$, Marc Schallenberg ${ }^{1}$

7

$8{ }^{1}$ Department of Zoology, University of Otago, P.O. Box 56, Dunedin, New Zealand

$9 \quad{ }^{2}$ BC3-Basque Centre for Climate Change, 48940, Leioa, Spain

$10 \quad{ }^{3}$ Leibniz-Institute of Freshwater Ecology and Inland Fisheries, Müggelseedamm 310, 12587

11 Berlin, Germany

13 *Author for correspondence:

14 Simone D. Langhans; $\underline{\text { simone.langhans@otago.ac.nz }}$

16 ACKNOWLEDGEMENTS

17 SDL has received funding from the European Union's Horizon 2020 research and innovation 18 programme under the grant agreement No. 642317, the Marie Skłodowska-Curie grant

19 agreement No. 748625, and from Hawke’s Bay Regional Council. SCJ acknowledges

20 funding for the "GLANCE" project (Global Change Effects in River Ecosystems; 01

21 LN1320A) through the German Federal Ministry of Education and Research (BMBF). We

22 thank Fabiola C. Rodríguez Estrada for graphical support, and two anonymous referees, the

23 editor J. Price and additional editors for comments that considerably improved the 24 manuscript. 
ABSTRACT

27 Freshwater ecosystems are essential to peoples' economic, cultural, and social wellbeing, yet

28 are still among the most threatened ecosystems on the planet. Consequently, a plethora of

29 recent regulations and policies seek to halt the loss of, restore, or safeguard freshwaters, their

30 biodiversity, and the ecosystem services they provide. Ecosystem-based management (EBM),

31 an approach that considers human society as an integral part of ecosystems, is increasingly

32 being promoted to help meet this challenge. EBM involves an overarching regulatory

33 framework and local solutions with trade-offs and compromises - factors that make decision

34 processes complex, but also provide the means for combining top-down regulation with

35 bottom-up priorities into collaborative management strategies. Although stakeholder

36 participation is encouraged in most modern freshwater management, community values are

37 often largely neglected. Here, we introduce a well-known participatory decision support

38 framework based on multi-criteria decision analysis (MCDA) to operationalize EBM and

39 promote community-inclusive decision-making in freshwater management. We explain the

40 different steps that this approach comprises which lead to the prioritisation of a management

41 strategy in a collaborative way. We also show how cultural values that inherently embed

42 strong links between the environment and people, can be used together with typical

43 ecological and socio-economic values. We illustrate the MCDA-based EBM-approach for

44 New Zealand, one of the few countries in which regional freshwater management is

45 mandated to uphold environmental quality standards, while safeguarding local community

46 values and ecosystem services. Finally, we discuss some of the challenges which are

47 increasingly emerging as a result of mandated community collaboration in environmental

48 management.

50 KEY WORDS: bottom-up, community buy-in, collaborative, indigenous values, Māori, 51 multi-criteria decision analysis, MCDA, top-down 


\section{INTRODUCTION}

53 Freshwater ecosystems are among the most threatened and modified environments on the

54 planet, with freshwater biodiversity decreasing more rapidly than in marine or terrestrial systems (Vörösmarty et al., 2010; WWF, 2016). Healthy freshwater ecosystems are essential for both maintaining biodiversity and for ensuring people's economic, cultural, and social wellbeing. Impacting these ecosystems has already led to, and will further increase, the loss of water-based ecosystem services (ES) people receive from them (Russi et al., 2013). Current national and international water and environmental regulations (e.g. the European Union's Water Framework Directive (WFD; 2000/60/EC), the EU 2020 Biodiversity Strategy (COM/2011/244 final) and the Convention on Biological Diversity) mandate the management of freshwater ecosystems in a way that acknowledges social-ecological interactions, rather than treating society and the environment as separate entities.

Ecosystem-based management (EBM) is increasingly proposed as an approach that can incorporate such interactions. There is no agreed-upon definition of EBM, but it can generally be understood as a collaborative-management (often referred to as co-management) approach intended to restore, enhance, and/or protect the resilience of an ecosystem so as to sustain or improve the flow of ES and to conserve biodiversity, while considering human society as an integral part of that ecosystem (Long, Charles, \& Stephenson, 2015).

Progressing from economic/environmentally-driven management to management that also considers social drivers and implications requires change. Governance modes and local and national policies have to shift from top-down regulation to more bottom-up, local decision-making structures, involving stakeholder entities interested in the management decisions. The WFD, for example, mandates each EU member state to plan freshwater improvement in river basin management plans (European Commission, 2012), which should be prepared and updated in participatory processes that inform and consult with interested stakeholder entities (European Commission, 2003). Similarly, the United States' Clean Water Act pursues the objective of maintaining and restoring aquatic ecological integrity and expects stakeholder participation to contribute to developing, revising, and enforcing regulations and management plans (Federal Water Pollution Control Act, 1948).

Despite such regulations, so far most management approaches have mainly focused on integrating top-down environmental and economic values, whereas cultural values and local knowledge have received little attention in practice (Daniel et al., 2012). Here we consider cultural values to be "non-material benefits people obtain from ecosystems through spiritual enrichment, cognitive development, reflection, recreation and aesthetic experience" 
(Robertson, 2004). Management decisions that relegate cultural values to an afterthought have been criticized by ecologists who perceive intrinsic values in nature (Redford \& Adams, 2009) and by theorists who are critical towards commodification of nature (Salmond, Tadaki, \& Gregory, 2014). Cultural values are often location-specific and, therefore, they may not be adequately considered by regionally- and nationally-mandated regulations. Consequently, we argue that future environmental decision making for freshwater management could greatly benefit from a more explicit and more structured incorporation of cultural community values than at present. How to effectively do this is the subject of this paper.

Examples of cultural values people relate to fresh waters can include swimming, boating, angling, feeling calmed, inspired, happy and/or energized when spending time at, on, or in, a freshwater body or experiencing its beauty etc. There are two main challenges when including cultural values in structured, analytical decision making. Firstly, many have argued that these values are incommensurate and not amenable to economic trade-offs (Miller, Tait, \& Saunders, 2015). For example, a management action may be seen as violating a deeply held principle and, therefore, the use of trade-offs is rejected, stalling progress in collaborative decision making. Secondly, cultural values are often difficult to articulate and quantify.

Many of the problems in natural resource management, including the development of community-inclusive freshwater EBM strategies, are so-called 'wicked problems' (Parrott, 2017). Problems are considered as 'wicked', if there is no single, optimal, or clear solution (sensu Rittel \& Webber (1973)) due to inherent competing or conflicting interests and 'different ways of knowing' (i.e. unrecognized contextual, methodological, and substantive differences among knowledge systems; sensu Brugnach \& Ingram (2012)). Consequently, in the absence of clearly structured and well-communicated processes, EBM can be co-opted (Duncan, 2013). This challenge can, however, be overcome with well-designed processes that are flexible, adaptive, and include scenario development and evaluation (Sterling et al., 2017). Hence, there is an urgent need for a support framework based on collaborative decisions that is (i) transparent, (ii) allows for stakeholders' ecological, socio-economic, and cultural values to be quantified and accounted for, (iii) allows for the concurrent consideration of top-down and bottom-up defined values, (iv) can mathematically test and compare outcomes of different management alternatives, and (v) can ultimately prioritise management actions with collective buy-in, (vi) while accounting for uncertainty. Statutory promotion of such a framework would assure its implementation and provide opportunities to further refine it. 
Here, we introduce Multi-Criteria Decision Analysis (MCDA) as a participatory structured decision support tool, and outline the ten iterative steps that can facilitate the formal development of a freshwater management plan. We then show how MCDA allows for

123 mixed collaboration, i.e. the concurrent inclusion of top-down environmental regulatory

124 limits as well as bottom-up, locally-defined community values and preferences. We show

125 how New Zealand's current approach to freshwater management is compatible with such a

126 framework. Finally, we discuss the potential of MCDA to benefit EBM by strengthening the prospects of mixed collaborative approaches.

\section{MULTI-CRITERIA DECISION ANALYSIS (MCDA)}

130 Multi-Criteria Decision Analysis (MCDA) is a generic term for a collection of theories and 131 approaches which offer support in complex decision situations facing multiple, conflicting 132 objectives and large uncertainty (Eisenführ, Weber, \& Langer, 2010). MCDA decomposes these complex decision situations into manageable parts to help systematically evaluate and prioritize management alternatives. Thereby, the relative importance of the goals of the decision situation is defined through weights that represent stakeholders' preferences. Management alternatives are evaluated and ranked based on their predicted consequences for each goal, incorporating trade-offs among these consequences and measures of uncertainty.

During the last decade, MCDA has gained popularity in helping with river management decisions. Reichert et al. (2015) proposed a conceptual framework for environmental decision support that employs the best available scientific knowledge to identify management alternatives with the highest likelihood to achieve ecological, economic, and societal goals. As a hypothetical case study they conducted a spatial river restoration prioritisation for a small river catchment in Switzerland. Comino et al. (2016) applied spatial MCDA to support policy and action definition for managing the Pellice river basin in Italy. Langhans et al. (2016) found that four MCDA-elements which are often simplified in river restoration assessments, due to time and/or resource constraints, do not reflect experts' opinions and should therefore be avoided in implementations. And most recently, Paillex et al. (2017) used MCDA to assess and compare the ecological quality of a restored and an unrestored river reach in Switzerland.

Among the range of different MCDA methodologies, multi-attribute value theory and multi-attribute utility theory (MAVT/MAUT) are increasingly used for environmental management (Reichert et al., 2015). A range of properties make these theories especially interesting for freshwater management (Paillex et al., 2017): (i) They are based on axioms of 
rational choice which is useful when decisions have to be justified (e.g., to the taxpayers or the public in general), (ii) they focus explicitly on the goals that should be achieved through the implementation of a management plan and not on the selection of the management action itself; (iii) new management actions can be included at any stage of the decision process without triggering a change in the ranking of the already included alternatives, (iv) they can consider uncertainties, for example of the environmental assessment, the prediction of the

\section{TEN STEPS TO A MCDA-DRIVEN EBM-FRESHWATER MANAGEMENT PLAN}

To facilitate communication with stakeholder entities, we have split the MCDA-process into ten discrete steps adapted from Reichert et al. (2015), as shown in Figure 1.

Steps A and B: Problem framing and stakeholder analysis. The first step in making an informed decision is to clearly define the problem, i.e. the main goal (Step A). For freshwater management this could involve identifying a management strategy for the respective freshwater system that considers regulations and has buy-in from the community. The relevant stakeholders who are to be involved in tackling the problem are then identified (Step B), possibly using snowball sampling. For freshwater management, relevant stakeholders could include representatives of the local government responsible for managing fresh waters, local indigenous groups, farmers, fishermen, agriculture industry, tourism, kayakers/canoers, yachting clubs, conservation groups, the local community, etc. Steps A and B can usually be prepared by the facilitator before the first stakeholder workshop. However, the details need to be confirmed (or reframed) and additional stakeholders might need to be identified during the initial workshop.

Step C and D: Identifying values, sub-goals, and attributes. Sub-goals are desired outcomes of the decision process (e.g. increased fish harvest from a lake) and are either derived from environmental directives (compulsory, environmental goals) or are identified by stakeholders, thereby reflecting their values (i.e. activities, uses, sources of value, or "things that matter"; Step C). The sub-goals can then be arranged, possibly divided into more specific sub-goals, and organized in a hierarchy $\left(\right.$ Step $\left.\mathrm{D}_{1}\right)$. The hierarchy should only contain distinct goals to avoid double counting. Additionally, the sub-goals should conform with preference independence, meaning that preferences for the level of one sub-goal can be specified independently of the level of other sub-goals (Eisenführ et al., 2010). The splitting of goals 
188 into sub-goals allows to identify and assign attributes to the most explicitly defined sub-goals 189 at the bottom of the hierarchy. Attributes are measurable system properties or indicators

190 (Steps $\mathrm{D}_{2}$ ). If a sub-goal is difficult to measure, a proxy attribute can be used. Steps C and D 191 can be undertaken with all stakeholders in a workshop format. Different techniques, such as 192 working with sticky-notes, can be used to make sure that all stakeholders have a voice in the 193 workshops. 


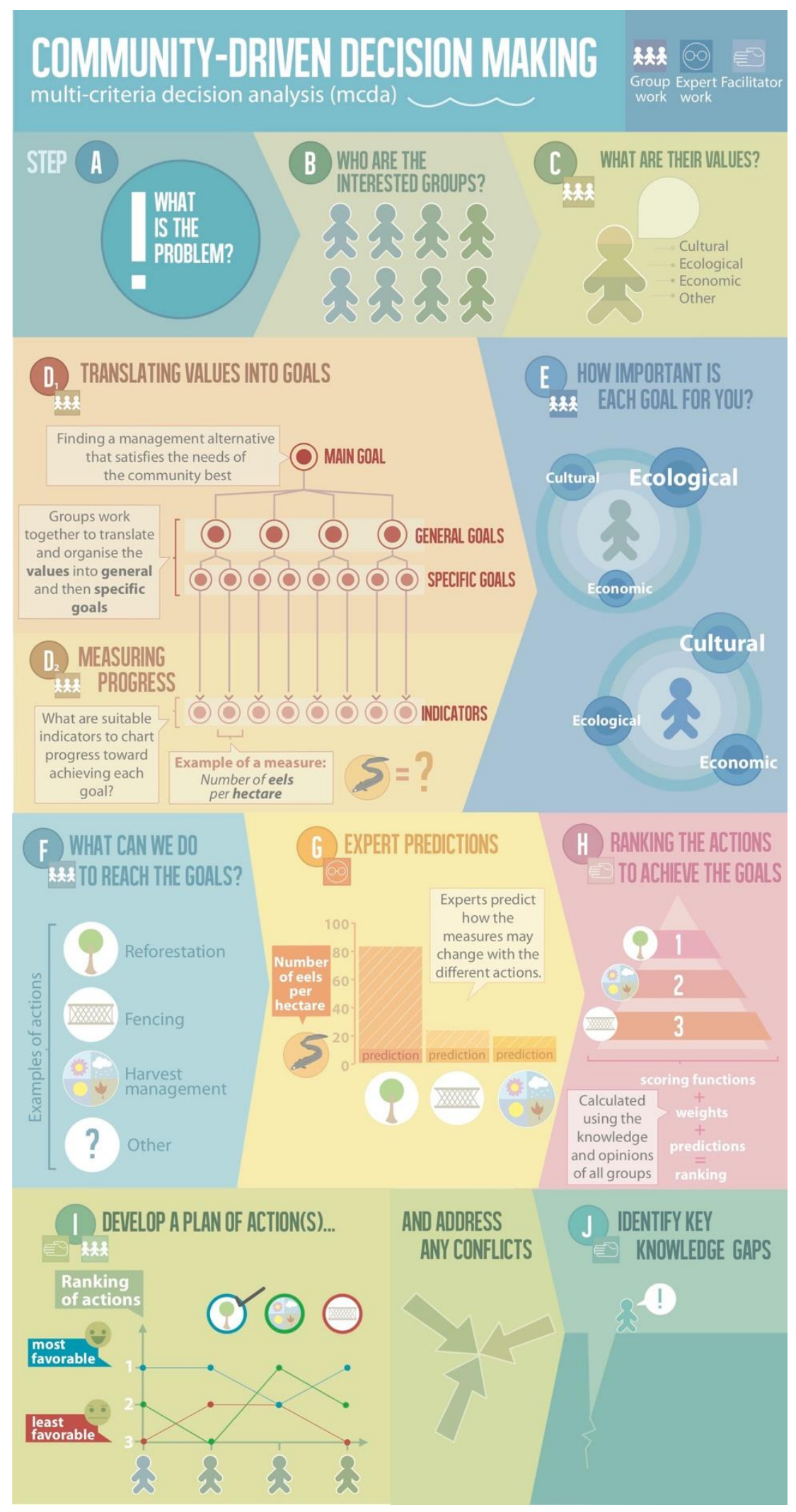

195 Figure 1. Infographic showing the ten different steps adopted in the community-driven

196 decision making process based on Multi-Criteria Decision Analysis (MCDA).

197 Step E: Quantifying value preferences and weights of goals. MCDA focuses on value

198 scores to prioritise management actions rather than asking stakeholders directly which action 
they prefer. Mathematically identifying priority actions can lead to surprising results. For example, when stakeholder's initial preferred management action does not end up being the best action to safeguard her/his priority values after prioritization is based on her/his quantified values. To be able to calculate the value scores based on all attributes, the identifying a scoring function for each attribute (Fig. 2). Scoring functions have a continuous scale of 0 to 1 on the $y$-axis and the considered range of the attribute on the $x$-axis $(0=$ no achievement, 1 = full achievement of the sub-goal). The functions can either be translated from already established assessment protocols, for example to conform with compulsory goals (see below). They can also be elicited from stakeholders in interviews, for example using the mid-value splitting method (Lienert, Koller, Konrad, McArdell, \& Schuwirth, 2011). Interviews should follow a strict protocol to minimize biases due to framing, availability, and social context (Burgman et al., 2011). The shape of each scoring function reflects the stakeholders' preferences on how each attribute relates to each sub-goal. Where multiple scoring functions are elicited for the same attribute for the same stakeholder group, and have to be pooled to represent a summary of this group's opinion, a variety of combination methods can be used (e.g., Stewart \& Quintana, 2018).

Scoring functions describe stakeholders' preferences regarding certain attribute outcomes, whereas utility functions describe preferences in relation to risky ones. Utility functions can either be directly elicited from stakeholders or converted from scoring functions after accounting for the stakeholders' attitudes towards risk (Dyer \& Sarin, 1982).

Stakeholders also define the relative importance of sub-goals by assigning weights to each of them. For example, a stakeholder might decide to give a high weight to one of the subgoals to indicate a preference for this goal. Assigning a weight of zero results in the exclusion of the respective sub-goal for the respective stakeholder. Again, this process is done by following a standardized protocol (Step E). A common method for the elicitation of weights is the (reverse) swing method explained in (Lienert et al., 2011). Where multiple weights for the same sub-goal for the same stakeholder group are identified, these can be combined using different weighting schemes to represent the group's opinion (Cooke, ElSaadany, \& Huang, 2008). 


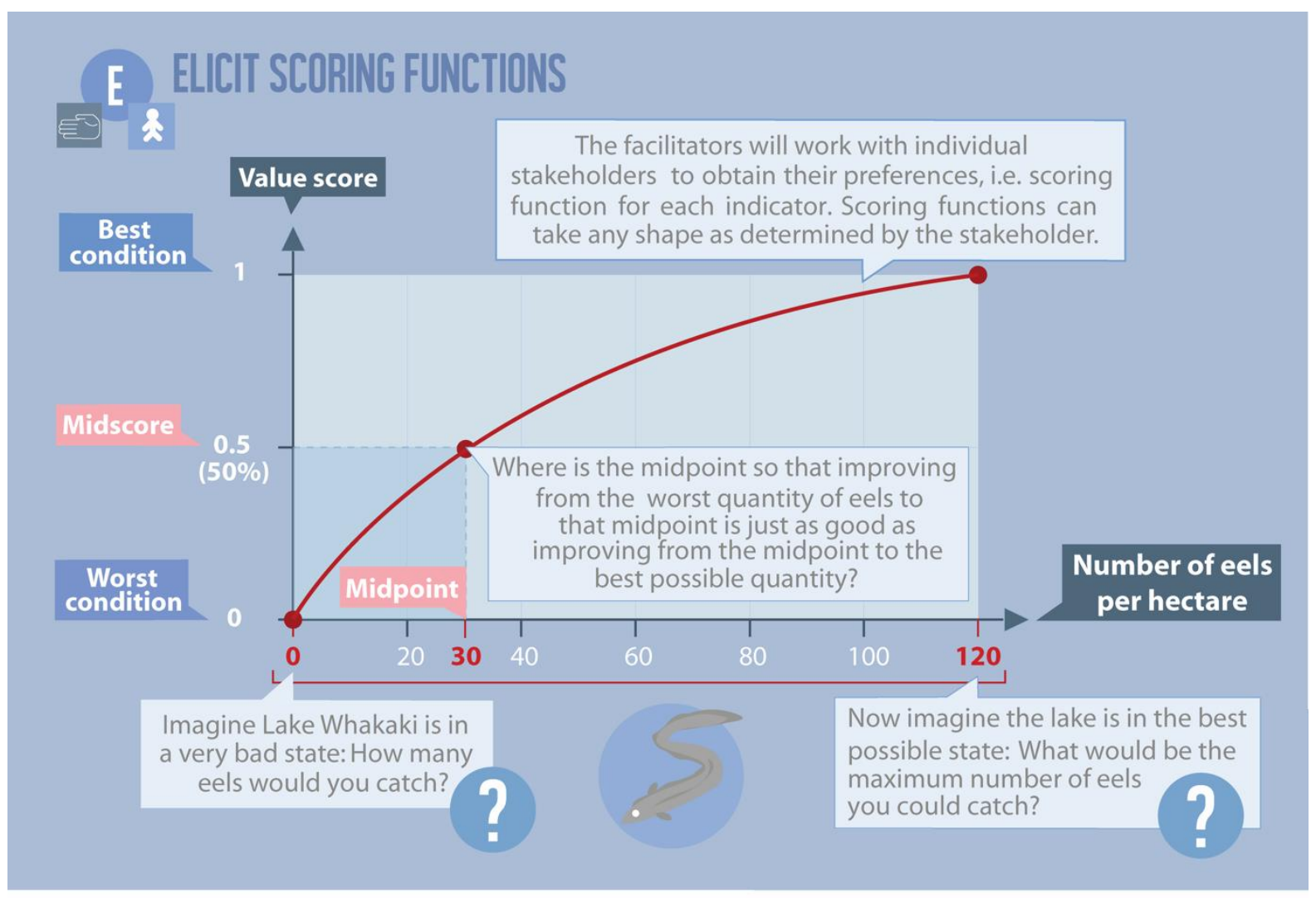

230 Figure 2. Example of the quantification of value preferences in the form of a scoring function. Scoring functions have a continuous scale from 0 to 1 on the $y$-axis and the considered range of the attribute in its original unit on the $\mathrm{x}$-axis $(0=$ no achievement, $1=$ full achievement of the goal). This step of the MCDA process is excluded from the main infographic, since it is the most complex one, requiring more detailed explanation.

Steps F and G: Identifying management actions and predicting outcomes for each alternative. Stakeholders identify potential management actions (Step F). How each attribute will change with each of the potential actions is then projected or predicted based on environmental system models or expert judgement, respectively (Step G).

\section{Step H: Combining steps $E$ and $G$ to calculate the value of each management action} for each stakeholder entity. Predicted attribute levels for each alternative management

247 Aggregating additively allows for compensation (i.e. a good value can, to some degree, 
compensate a bad one when aggregated), and therefore increases the statistical significance of the results. Sub-goals that are complementary to each other (such as those often found at higher levels of the hierarchy) should be aggregated with a mixture of additive and minimum aggregation (also called worst case or one-out, all-out) to allow for some compensation but yet still penalize for sub-goals with a very low score (Langhans et al., 2014).

\section{Step I: Ranking the alternatives for each stakeholder, addressing conflicts, and} finding a commonly approved solution. For each stakeholder the actions are ranked according to decreasing overall score. The stakeholder-specific rankings of the potential actions are then discussed among all participating parties in a workshop. At this stage, the insights gained by going together through the described process facilitates agreement on one or more of the management actions, or the identification of a new, compromise solution to the management problem.

Step J: Identifying key knowledge gaps. Having performed the previous nine steps, knowledge gaps will have become apparent. Any new knowledge gained can be included in

\section{MCDA FOR NEW ZEALANDS’ FRESHWATER MANAGEMENT}

\section{Background to freshwater management in NZ}

In 1991, New Zealand (NZ) adopted an integrated approach to freshwater management (Resource Management Act, 1991), which has recently been further developed in the National Policy Statement for Freshwater Management (NPS-FM; NZ Ministry for the Environment, 2017). Compared to the previous approach, the NPS-FM recognizes diverse elements of freshwater use that contribute to wellbeing in society. Hence, to conform with the NPS-FM, the next generation of management plans must consider values that are important to the community as well as compulsory water quality and health limits. Consequently, future NZ freshwater management plans will combine governmental and community objectives. Currently, freshwater management plans are developed for each of the 16 regions in NZ.

$\mathrm{NZ}$ is one of the many countries around the world where fresh waters are of major importance to indigenous people. To Māori water is a tupuna (ancestor), which is why it is considered a taonga (treasure). In addition, waterways provide resources for cultural products such as mahinga kai, which is a Māori term for traditional food and resource gathering (Tipa \& Teirney, 2003). Hence, fresh water is crucial in maintaining Māori traditions and knowledge (Harmsworth, Young, Walker, Clapcott, \& James, 2011). Following the international trend to increasingly include indigenous communities as active participants in 
282 environmental decision making, NZ's local and central governments are eager to engage with

283 Māori groups (iwi/ hapū) in freshwater management planning processes (Harmsworth et al.,

284 2016). Actually, legal requirement to do so is given by the Treaty of Waitangi, which is the

285 foundation document of Māori rights. The NPS-FM takes up the Treaty mandate by

286 specifically considering Māori’s water values separately from other community values (see

287 below) and by emphasizing on iwi/hapū to play a key part as partners in the participatory

288 freshwater-management planning-process.

290 Applying steps D and E of the MCDA-framework to the NPS-FM

291 The NPS-FM sets out 13 national values and uses for fresh water. Two of them are

292 compulsory when developing a freshwater management plan: (i) ecosystem health and (ii)

293 public health and recreation (NZ Ministry for the Environment 2017). For the eleven

294 remaining national values, the goals, attributes, and their measurement methods will be

295 defined by the local community. Figure 3 shows how the compulsory national values and the

296 non-compulsory values described in the NPS-FM could be structured hierarchically

297 according to step D of the MCDA process (Fig. 1). Additional goals and corresponding

298 attributes identified by iwi and stakeholders during the MCDA-process can be included in the

299 hierarchy either as a new high level goal, or under one of the existing branches of the

300 hierarchy.

301 The NPS-FM defines attributes for the compulsory values/goals: Seven attributes to

302 measure ecosystem health ('phytoplankton', 'total Nitrogen', 'total Phosphorus',

303 'periphyton', 'Nitrate', 'Ammonia', 'dissolved Oxygen') and two attributes ( 'cyanobacteria',

304 'E. coli') to assess whether water quality does not harm people's health, when they use water

305 bodies for recreational purposes (Fig. 3). 


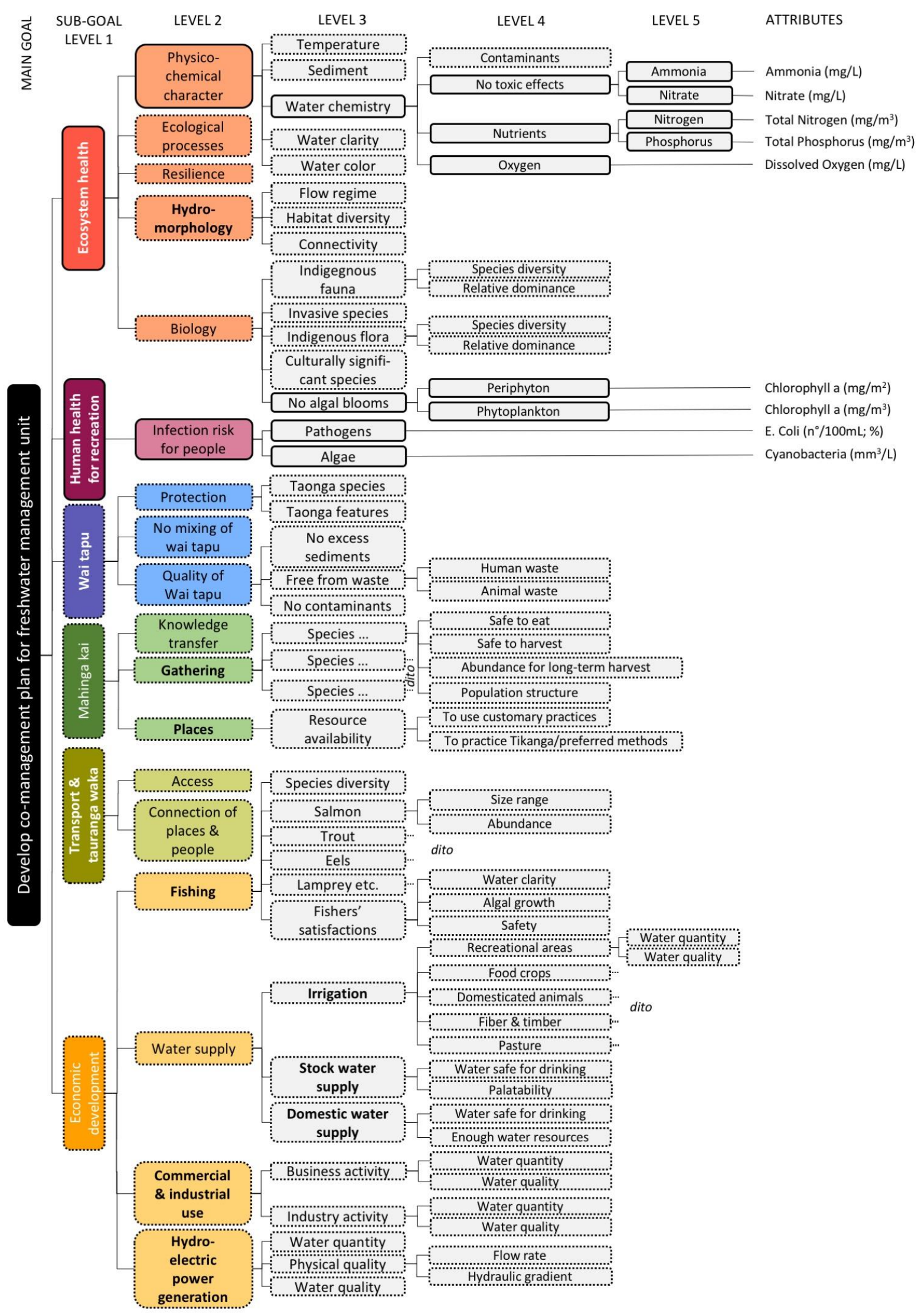

Figure 3. Objectives hierarchy of compulsory national values (in solid boxes) and other 
309 freshwater co-management strategies in New Zealand. The sub-goals in bold are the headings

310 of the 13 value categories outlined in the National Policy Statement for Freshwater

311 Management (NPS-FM).

The NPS-FM provides quality bands for the nine attributes that can be used to establish scoring functions for each of the attributes (c.f., step E, Fig. 2; see Langhans et al. (2013) for a complete description of how to transfer quality assessments into scoring functions). For the attributes 'phytoplankton', 'total Nitrogen', 'total Phosphorus', 'periphyton', Nitrate', 'Ammonia', 'dissolved Oxygen' and 'cyanobacteria' (Fig. 4 a-h), the NPS-FM defines four quality bands (A-D). For the attribute 'E. coli', which is a proxy attribute for assessing swimmability of fresh waters (Fig. $4 \mathrm{i}$ and j), five quality bands were developed. Following other national water quality assessment protocols that use scoring functions (Niederberger et al., 2016), we assumed that each quality band stands for the same increase in quality and, therefore, in freshwater value $(0,0.25,0.5,0.75,1.0$ for $\mathrm{a}-\mathrm{h}$; and $0,0.2,0.4,0.6,0.8,1$ for $\mathrm{i}$ and $\mathrm{j}$ ). According to the NPS-FM, the national bottom line lies between the quality bands $\mathrm{C}$ and $\mathrm{D}$ (band $\mathrm{D}$ is deemed unacceptable), which consequently corresponds to a value of $\leq 0.25$ (a-h), while there is no simple national bottom line set for the attribute 'swimmability'.

Minimum values $(y=0)$ of a scoring function represent the worst possible condition of the attribute in the respective freshwater system, while maximum values $(y=1)$ represent the best possible condition (Fig. 4). Since minimum and maximum values are not defined in the NPS-FM, they were defined for this exercise by an expert with extensive experience in NZ freshwater ecology (M. Schallenberg, University of Otago). However, the definition of minimum and maximum values for the compulsory national values as well as assigning values to the quality bands should be verified and confirmed more generally before using the MCDA process to design freshwater management plans. Scoring functions for attributes that measure the non-mandatory values have to be elicited from stakeholders. 

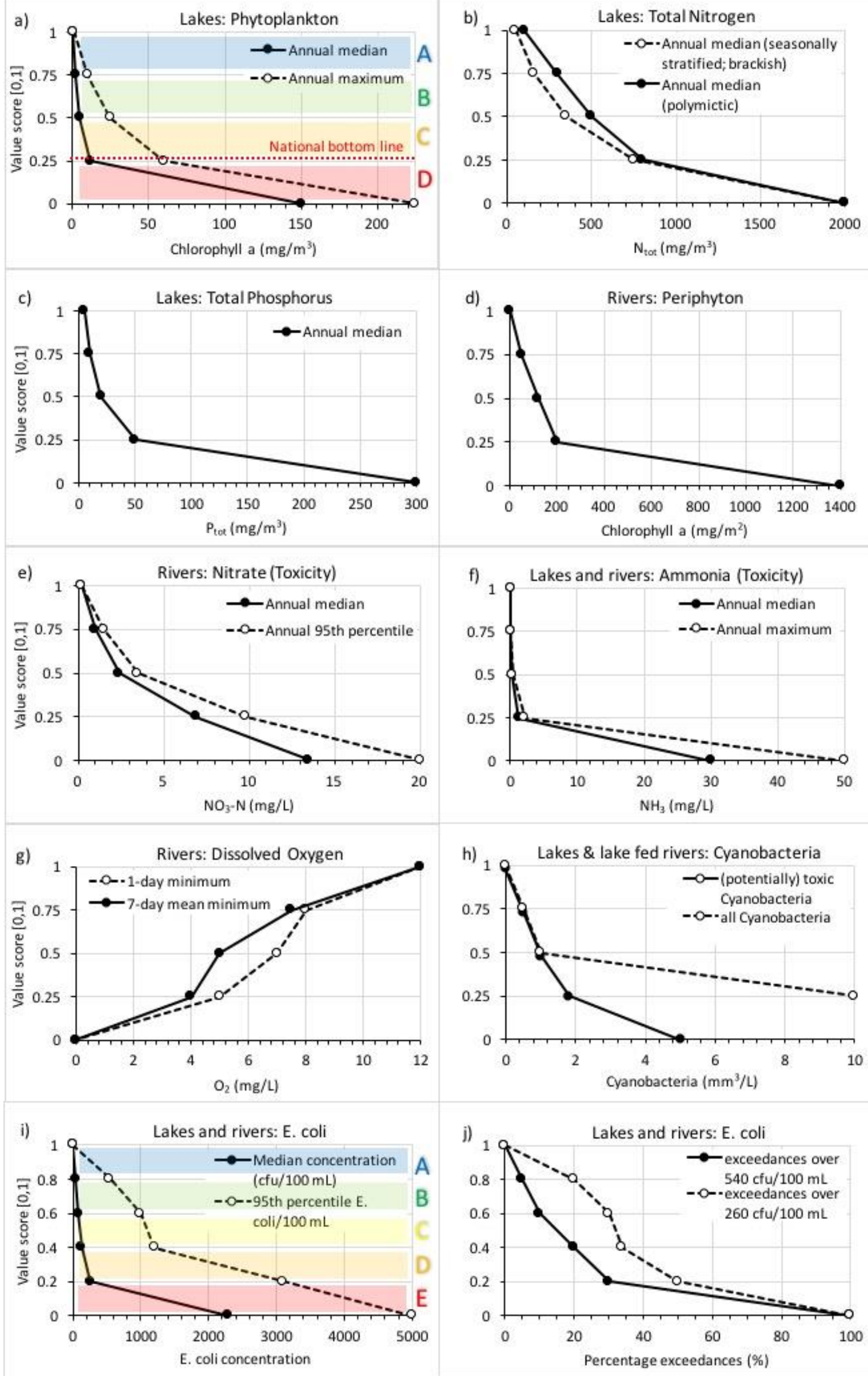
Figure 4. Scoring functions for the attributes that quantify the compulsory national values translated from the National Policy Statement for Freshwater Management (NPS-FM).

\section{The role of experts in the MCDA process}

The NPS-FM aims to maintain all freshwater ecosystems in a healthy ecological state and to restore those that are degraded from such a state (NZ Ministry for the Environment, 2017). For iwi and stakeholders, it can be difficult to identify all relevant sub-goals and measurable system attributes as well as to quantify the degree of fulfillment of the sub-goals as a function of the attributes (Reichert et al., 2015). Therefore, Reichert et al. recommend that this part of the hierarchy is elicited from experts (i.e. freshwater ecologists) or translated from existing procedures, as we show here using the quality assessments given in the NPS-FM for the different attributes. Where experts are relied on to define sub-goals, attributes, and scoring functions, these elements should be carefully explained to the participating iwi and stakeholders, so that they can assign weights to all sub-goals in an informed way.

$$
\text { Another important role experts can play in the MCDA process is to help iwi and }
$$
stakeholders identifying potential management alternatives. Iwi and stakeholders might not be aware of, or up-to-date with, the latest available technologies and management actions. Experts also develop and apply conceptual and quantitative models to project the consequences of the potential management actions. Where sufficient data to construct quantitative models is unavailable, experts' system knowledge can be elicited to predict the potential effects of the management alternatives (Reichert et al., 2015).

\section{8 stakeholders build informed preferences.}


EBM is complex, because it involves community participation and local solutions with tradeoffs and compromises within an overarching regulatory framework (Kiker, Bridges,

373 Varghese, Seager, \& Linkov, 2005). This complexity also reflects the need to simultaneously 374 integrate mandated freshwater values with locally-defined limits and community priorities, as 375 it is currently required for freshwater management in NZ (NZ Ministry for the Environment 376 2017). Combining top-down regulations with bottom-up participation in a community-

377 inclusive approach has been suggested to be the optimal approach to deal with environmental management challenges (Khadka \& Vacik, 2012). Therefore, an MCDA-based decision support framework has the potential to facilitate the development of freshwater EBM in multiple ways:

- MCDA entails a formal process with a long track record in social science. It can therefore

- MCDA is mostly community-driven, assisted by experts informing the decision support process, and facilitators leading the community through the different steps. It is important to note that facilitators are neutral and do not contribute their values and preferences to the process.

- The different steps of the MCDA process are transparent; the model structure, shapes of value/utility functions and weightings given to the input data are clearly observable to stakeholders. This understanding creates trust in the decision recommendation and promotes commitment in implementing management actions. Moreover, transparency fosters learning by, for example, allowing the collaborative exploration of how changes in input values and preferences can influence the prioritization of the management actions (Salo \& Hämäläinen, 2010).

- The discrete steps in the decision support process can easily be iterated when new knowledge is available. It is therefore compliant with the concept of adaptive management.

- During the process of developing an MCDA-based EBM plan, the integrities of both subjective freshwater values and objective freshwater system knowledge are maintained. These components are only combined at the end of the process, when prioritising the optimal management actions for the involved entities. Hence, the prioritisation is based on a purely mathematical calculation and is, therefore, immune to power dynamics among stakeholders. 
- The process embodies information sharing and communication across iwi/ hapū, stakeholders and decision makers during multiple workshops, which supports the convergence of opinion and ideas. Social learning is the recognition that people learn through active adaptation of their existing knowledge in response to their experiences with other people and their environment (Allen et al., 2011). Hence, it is likely that information sharing is also beneficial for fostering compromise solutions in freshwater management, where multiple ways of knowing and multiple types of knowledge must be incorporated into the decision making process.

- The prioritisation of management actions is the focus of the MCDA-based EBM approach. Outputs of the EBM-MCDA process can, therefore, directly lead to the development of catchment management plans with high levels of community buy-in.

- The MCDA-based EBM approach can consider both values that have been mandated (e.g.,

421 There is ample literature discussing potential challenges that might need to be tackled when National water quality guidelines) and community-defined, bottom-up values, including difficult-to-quantify, cultural values. In addition, MCDA allows the consideration of indigenous values such as for example mahinga kai as a value for food provisioning separately from mahinga kai as a cultural value. using MCDA (e.g., Reichert et al., 2015). Common challenges, which can also not be overcome when applying best practice, include i) the time-consuming nature of working through the process, especially the elicitation of scoring functions, ii) traditional decision makers (e.g., environmental authorities) may not be interested in participating in the process or in providing information to it, and iii) the MCDA process does not necessarily identify a single, best management action for all stakeholders. It is up to stakeholders and decision makers as to whether the outcome of the process leads to an overall agreement on one of the actions or to a compromise solution. The process can only be successful, if participants are willing to collaborate in a consensus-seeking spirit. Besides these challenges, we identified four additional ones that might be specifically relevant for NZ, which we elaborate on below.

\section{Finding agreement within stakeholder groups}

MCDA processes commonly start with the identification of different stakeholder groups that have an interest in, or are affected by, the decision making. Doing so is based on the 
assumption that all individuals of such a group have the same, or very similar, preferences. Involving the community in freshwater management planning leads to the challenge that 'the community' is likely not a stakeholder group with homogenous preferences, but one with interpersonal differences and goal incongruities (Matsatsinis, Grigoroudis, \& Samaras, 2005). A way forward in this case is to apply factor or cluster analysis to base the assignment of 'the community' (as well as all other participants) to specific stakeholder groups based on their preferences, i.e. how they weight the different sub-goals (Spath, 1980). Examples of stakeholder groups emerging from a cluster analysis could be 'spiritualists ' (people of the community who believe the freshwater system should be in a healthy condition because of ethical reasons), 'sustainable users ' (people who have an economic interest in fresh water and want to use it sustainably), 'fresh water recreationists ' (people who want the freshwater system to be in a good enough status and equipped with the necessary infrastructure to enjoy a freshwater-based recreational activity) and so on.

\section{Power sharing}

MCDA is based on the assertion that all parties (whether they are in positions of power to make decisions about the environment or not) that are interested in the decision problem should be part of the decision making process. Where there are power imbalances, it could be decided a priori that decision making will be shared equitably among all of the parties (i.e., strong co-management, sensu Taiepa et al. (1997)). This form of co-management is distinct from a process where iwi and stakeholders develop a management plan that serves as a 467 and policy. recommendation to the decision maker. A major risk of such a non-equitable decision approach is the creation of consultation fatigue, if the recommendation is not, or not enough, considered by the decision authority (Reed, 2008). Poor personal reward or little capacity to influence decisions may result in iwi and stakeholders not being willing to participate in future projects. Hence to make the collaboration process as successful as possible and to increase the likelihood that a co-developed management plan is implemented successfully, the decision makers (e.g., the environmental authorities) should participate in good faith in the MCDA process. This challenge might be aggravated in regions where iwi/hapu find their partner role in the collaboration process not being appropriately acknowledged and protected, despite the Treaty of Waitangi and its principles being embraced in current NZ legislation 

Social learning 
470 Inclusive workshops are crucial to bring people with different preferences together, so they

471 can share their values and opinions. Doing so can lead to a learning process which may

472 ultimately facilitate compromise solutions (Brymer, Wulfhorst, \& Brunson, 2018). In NZ, a

473 parallel process to freshwater management where Māori are given the space to carry out their

474 own identification of values, weights, and attributes - reflecting a Treaty partnership process -

475 has been advocated (Robb, Harmsworth, \& Awatere, 2015). The application of such a

476 parallel process will likely reduce the benefits of social learning as compared to an inclusive

477 MCDA-based process. However, MCDA is flexible enough to accommodate such structuring

478 of the decision-making process, where otherwise freshwater management planning would be

479 stalled. The focus of this study is on policy development.

Trust building

482 Like any other participatory decision and planning process, an MCDA-based decision 483 process heavily relies on forming trusted relationships (Heldt et al., 2016). In NZ, this trust needs to be built between the stakeholder groups and the institutions with the authority to make decisions which affect freshwater management (i.e. the Crown (NZ Government) and/or Regional Councils) but also among the various stakeholder groups. Additionally, the quality of the relationships between iwi/hapū and all the other participants will likely play a significant role in the success of the participation process and, therefore, in developing a comanagement plan (Harmsworth \& Awatere, 2013).

\section{CONCLUSIONS}

There is an increasing awareness that environmental decisions, and therewith biological and cultural diversity, can benefit from the inputs of local and indigenous knowledge (Gavin et al., 2015) as well as from community buy-in. NZ's NPS-FM is therefore consistent with international regulations and initiatives, which increasingly mandate community involvement in environmental management. However, the implementation of processes to achieve this has lacked guidance and consistency among NZ Regional Councils, which must implement the policies. The MCDA-based approach we propose here is a helpful tool to facilitate greater community involvement in NZ's freshwater management and to potentially guide policy development. Although we applied it to a NZ case study, this approach has general applicability for including community preferences in environment decision making. 


\section{REFERENCES}

Allen, W., Fenemor, A., Kilvington, M., Harmsworth, G., Young, R. G., Deans, N., . . Smith, R. (2011). Building collaboration and learning in integrated catchment management: the importance of social process and multiple engagement approaches. New Zealand Journal of Marine and Freshwater Research, 45, 525-539.

Brugnach, M., \& Ingram, H. (2012). Ambiguity: the challenge of knowing and deciding together. Environmental Science \& Policy, 15, 60-71.

Brymer, A. L. B., Wulfhorst, J. D., \& Brunson, M. W. (2018). Analyzing stakeholders' workshop dialogue for evidence of social learning. Ecology and Society, 23(1):42.

Burgman, M., Carr, A., Godden, L., Gregory, R., McBride, M., Flander, L., \& Maguire, L. (2011). Redefining expertise and improving ecological judgment. Conservation Letters, 4, 81-87.

Comino, E., Bottero, M., Pomarico, S., \& Rosso, M. (2016). The combined use of spatial multicriteria evaluation and stakeholders analysis for supporting the ecological planning of a river basin. Land Use Policy, 58, 183-195.

Cooke, R. M., ElSaadany, S., \& Huang, X. Z. (2008). On the performance of social network and likelihood-based expert weighting schemes. Reliability Engineering \& System Safety, 93, 745-756.

Daniel, T. C., Muhar, A., Arnberger, A., Aznar, O., Boyd, J. W., Chan, K. M. A., . . von der Dunkj, A. (2012). Contributions of cultural services to the ecosystem services agenda. Proceedings of the National Academy of Sciences of the United States of America, $109,8812-8819$.

Duncan, R. (2013). Converting community knowledge into catchment nutrient limits: a constructivist analysis of a New Zealand collaborative approach to water management. Nature + Culture, 8, 205-225.

Duncan, R. (2016). Ways of knowing - out-of-sync or incompatible? Framing water quality and farmers' encounters with science in the regulation of non-point source pollution in the Canterbury region of New Zealand. Environmental Science \& Policy, 55, 151157.

Dyer, J. S., \& Sarin, R. K. (1982). Relative risk-aversion. Management Science, 28, 875-886. Eisenführ, F., Weber, M., \& Langer, T. (2010). Rational Decision Making. Berlin, Heidelberg: Springer-Verlag. 
European Commission. (2003). Common Implementation Strategy for the Water Framework Directive (2000/60/EC). Guidance Document No. 8 on public participation in relation to the Water Framework Directiv. Brussels, Belgium: European Commission. European Commission. (2012). Report from the commission to the European parliament and the council on the Implementation of the Water Framework Directive (2000/60/EC). River Basin Management Plans. Brussels, Belgium: European Commission.

Gavin, M. C., McCarter, J., Mead, A., Berkes, F., Stepp, J. R., Peterson, D., \& Tang, R. F. (2015). Defining biocultural approaches to conservation. Trends in Ecology \& Evolution, 30, 140-145.

Harmsworth, G., \& Awatere, S. (2013). Using mātauranga Māori to inform freshwater management. Policy Brief No. 7. Manaaki Whenua: Landcare Research.

Harmsworth, G., Awatere, S., \& Robb, M. (2016). Indigenous Maori values and perspectives to inform freshwater management in Aotearoa-New Zealand. Ecology and Society, 21(4).

Harmsworth, G. R., Young, R. G., Walker, D., Clapcott, J. E., \& James, T. (2011). Linkages between cultural and scientific indicators of river and stream health. New Zealand Journal of Marine and Freshwater Research, 45, 423-436.

Heldt, S., Budryte, P., Ingensiep, H. W., Teichgraber, B., Schneider, U., \& Denecke, M. (2016). Social pitfalls for river restoration: How public participation uncovers problems with public acceptance. Environmental Earth Sciences, 75:1053.

Khadka, C., \& Vacik, H. (2012). Comparing a top-down and bottom-up approach in the identification of criteria and indicators for sustainable community forest management in Nepal. Forestry, 85, 145-158.

Kiker, G. A., Bridges, T. S., Varghese, A., Seager, T. P., \& Linkov, I. (2005). Application of multicriteria decision analysis in environmental decision making. Integrated Environmental Assessment and Management, 1, 95-108.

Langhans, S. D., \& Lienert, J. (2016). Four common simplifications of multi-criteria decision analysis do not hold for river rehabilitation. Plos One, 11(3):e0150695.

Langhans, S. D., Lienert, J., Schuwirth, N., \& Reichert, P. (2013). How to make river assessments comparable: A demonstration for hydromorphology. Ecological Indicators, 32, 264-275.

Langhans, S. D., Schuwirth, N., \& Reichert, P. (2014). The method matters: guide to indicator aggregation in ecological assessments. Ecological Indicators, 45, 494-507. 
Lienert, J., Koller, M., Konrad, J., McArdell, C. S., \& Schuwirth, N. (2011). Multiple-Criteria Decision Analysis Reveals High Stakeholder Preference to Remove Pharmaceuticals from Hospital Wastewater. Environmental Science \& Technology, 45, 3848-3857.

Long, R. D., Charles, A., \& Stephenson, R. L. (2015). Key principles of marine ecosystembased management. Marine Policy, 57, 53-60.

Matsatsinis, N., Grigoroudis, E., \& Samaras, A. (2005). Aggregation and disaggregation of preferences for collective decision-making. Group Decision and Negotiation, 14, $217-$ 232.

Miller, S., Tait, P., \& Saunders, C. (2015). Estimating indigenous cultural values of freshwater: A choice experiment approach to Maori values in New Zealand. Ecological Economics, 118, 207-214.

Niederberger, K., Rey, P., Reichert, P., Schlosser, J., Helg, U., Haertel-Borer, S., \& Binderheim, E. (2016). Methoden zur Untersuchung und Beurteilung der Seen. Modul: Oekomorphologie Seeufer. Modul: Ökomorphologie Seeufer. UmweltVollzug Nr. 1632. Bern: Bundesamt für Umwelt.

Nielsen, H. O., Frederiksen, P., Saarikoski, H., Rytkonen, A. M., \& Pedersen, A. B. (2013). How different institutional arrangements promote integrated river basin management. Evidence from the Baltic Sea Region. Land Use Policy, 30, 437-445.

NZ Ministry for the Environment (2017) National Policy Statement for Freshwater Management. Updated August 2017 to incorporate amendments from the National Policy Statement for Freshwater Amendment Order 2017.

Paillex, A., Schuwirth, N., Lorenz, A. W., Januschke, K., Peter, A., \& Reichert, P. (2017). Integrating and extending ecological river assessment: Concept and test with two restoration projects. Ecological Indicators, 72, 131-141.

Parrott, L. (2017). The modelling spiral for solving 'wicked' environmental problems: guidance for stakeholder involvement and collaborative model development. Methods in Ecology and Evolution, 8, 1005-1011.

Redford, K. H., \& Adams, W. M. (2009). Payment for ecosystem services and the challenge of saving nature. Conservation Biology, 23, 785-787.

Reed, M. S. (2008). Stakeholder participation for environmental management: A literature review. Biological Conservation, 141, 2417-2431.

Reichert, P., Langhans, S. D., Lienert, J., \& Schuwirth, N. (2015). The conceptual foundation of environmental decision support. Journal of Environmental Management, 154, 316332. 
Resource Management Act. (1991). New Zealand Government, Wellington. http://www.legislation.govt.nz/act/public/1991/0069/211.0/DLM230265.html

Rittel, H. W. J., \& Webber, M. M. (1973). Dilemmas in a general theory of planning. Policy Sciences, 4, 155-169.

Robb, M., Harmsworth, G., \& Awatere, S. (2015). Maori values and perspectives to inform collaborative processes and planning for freshwater management. Hamilton, New Zealand: Landcare Research.

Robertson, M. M. (2004). The neoliberalization of ecosystem services: wetland mitigation banking and problems in environmental. Geoforum, 35, 361-373.

Russi, D., ten Brink, P., Farmer, A., Badura, T., Coates, D., Förster, J., . . Davidson, N. (2013). The Economics of Ecosystems and Biodiversity for Water and Wetlands. London and Brussels: IEEP, Gland: Ramsar Secretariat.

Salmond, A., Tadaki, M., \& Gregory, T. (2014). Enacting new freshwater geographies: Te Awaroa and the transformative imagination. New Zealand Geographer, 70, 47-55.

Salo, A., \& Hämäläinen, R. P. (2010). Multicriteria decision analysis in group decision processes. In Kilgour, D. M., \& Eden, C. (Eds.), Handbook of group decision and negotiation (pp. 269-283), Dordrecht: Springer.

Spath, H. (1980). Cluster analysis algorithms for data reduction and classification of objects. Chichester: Ellis Horwood Limited.

Sterling, E. J., Betley, E., Sigouin, A., Gomez, A., Toomey, A., Cullman, G., . . . Porzecanski, A. L. (2017). Assessing the evidence for stakeholder engagement in biodiversity conservation. Biological Conservation, 209, 159-171.

Stewart, R. T., \& Quintana, I. O. (2018). Probabilistic opinion pooling with imprecise probabilities. Journal of Philosophical Logic, 47, 17-45.

Taiepa, T., Lyver, P., Horsley, P., Davis, J., Bragg, M., \& Moller, H. (1997). Co-management of New-Zealand's conservation estate by Maori and Pakeha: a review. Environmental Conservation, 24, 236-250.

Tipa, G., \& Teirney, L. (2003). A cultural health index for streams and waterways: indicators for recognising and expressing cultural values. Technical Paper ME number 475. Wellington, New Zealand: Ministry for the Environment.

Federal Water Pollution Control Act. (1948). 33 U.S.C $§ § 1251$ et seq. U.S. Environmental Protection Agency. 
633 Vörösmarty, C. J., McIntyre, P. B., Gessner, M. O., Dudgeon, D., Prusevich, A., Green, P., . . 634 . Davies, P. M. (2010). Global threats to human water security and river biodiversity. $635 \quad$ Nature, $467,555-561$.

636 WWF. (2016). Living Planet Report 2016. Risk and resilience in a new era. Gland, 637 Switzerland: WWF International. 